\title{
Measurement of low-signal suppression and other parameters of the forced-choice psychometric function
}

\author{
PHILLIP L. EMERSON 1 \\ MENTAL HEALTH RESEARCH INSTITUTE, UNIVERSITY OF MICHIGAN ${ }^{2}$
}

\begin{abstract}
Empirical forced-choice psychometric functions often depart in shape from those based on the theory of normal sensory-excitation distributions and optimal decision processes. The typical departure is that the detectability of small signals is too low compared to that of high signals. Some possible explanations were reviewed and it was emphasized that the source of the effect is not known with certainty. An extra parameter resembling a threshold was introduced for the measurement of the effect, and a method of moments was proposed for estimating the two parameters of the resulting two-alternative forced-choice psychometric function. The method was tried out with success in the task of detecting a 0.1 -sec luminance increment to a steady adapting luminance of a large visual target. There were large amounts of low-signal suppression, indicating the advisability of reviewing some current psychophysical practices.
\end{abstract}

Low-signal suppression, so-called by Swets (1961), is defined in the context of the assumption of normal sensory-excitation distributions and the theory of optimal signal detection. The effect is often seen unambiguously in psychometric functions based on data from the symmetric two-aiternative forced-choice (S2AFC) task where the signal to be detected is a small stimulus energy increment to a relatively high background level. The S2AFC task is characterized by the presentation of two stimulus samples, only one of which contains the signal to be detected. It is symmetrical in the sense that either sample is equally likely to contain the signal. The S2AFC psychometric function then is the probability of correctly choosing the signal sample, as a function of some measure of signal magnitude. The ideal detector coupled to a Gaussian input channel of the simplest kind gives rise to a S2AFC psychometric function which is the upper half of the normal distribution function (Swets et al, 1961) which is monotonic increasing with a monotonic decreasing slope. Empirical S2AFC psychometric functions, on the other hand, often have slopes that increase to a maximum before beginning the monotonic decline with increasing signal strength, and this is what we will mean by "low-signal suppression." Other verbal descriptions of the effect (Swets, 1964, p. 132; Green, 1960, p. 130) differ from this in detail because it has been common in acoustical detection work to plot psychometric functions on a logarithmic transformation of signal energy. This transformation translates the origin to $-\infty$ which is unsuitable for the statistical techniques used here.

The main hypothesis as to the source of low-signal suppression has been that the $S$ has less than perfect knowledge of the signal to be detected, even when the signal is fixed and specified. Green and Swets $(1966$, Chap. 6, 7) have succeeded in formulations and numerical illustrations of the effects of several possible kinds of signal uncertainty on the S2AFC psychometric functions for ideal detectors, and in many cases the result is that the detectabilities of small signals are decreased relative to those of large signals. This does not prove that observed low-signal suppression is totally explainable on the basis of signal uncertainty, and indeed Green (1960) obtained the effect in a case where it seems doubtful that any of the signal-uncertainty formulations would be reasonable. There seems to be enough doubt to justify empirical parametric studies of the effect, since it is of great practical importance for psychophysical techniques which are based on an assumption as to the form of the psychometric function.

Although there will be an occasion to discuss another possible source of low-signal suppression in relation to the data to be reported here, we will attempt to develop a simple method of study which is somewhat neutral in regard to competing hypotheses. The general approach is to first assume that low-signal suppression is quantifiable in the sense that it may occur to greater or lesser degrees under different experimental conditions. When there is none, then the S2AFC psychometric function should be well approximated by the upper half of the normal distribution function as implied by the model of a Gaussian channel and an ideal detector, and the quantity should correspond to the degree of departure of the observed psychometric function from that of the ideal detector. These properties seem to be captured by the introduction of an extra parameter which might be interpreted as a threshold. Assume that the sensory channel is Gaussian in the sense that the difference between signal and no-signal excitations is distributed normally with unit variance and a mean of $x=d^{2} / \sqrt{2}$. Assume then in contrast to the ideal detector, that there is a difference threshold such that no difference is registered when the actual standardized difference between signal and no-signal excitations is within the threshold interval, $-\overline{\mathrm{c}, \mathrm{c}}$. Either choice is as good as the other when no difference is registered, and the probability of correct response is then $1 / 2$. Let $\Phi(\cdot)$ be the normal distribution function. Then the probability that no difference is registered is the area between the points $-c$ and $c$ of the standard normal distribution whose mean is $\mathrm{x}$, or $\Phi(\mathrm{c}-\mathrm{x})-\Phi(-\mathrm{c}-\mathrm{x})$. The joint probability of an observed difference and a correct response is the area above the point $c$, or $\Phi(x-c)$. Hence the total marginal probability of correct response, the psychometric function, is $1 / 2[\Phi(\mathrm{x}-\mathrm{c})+\Phi(\mathrm{x}+\mathrm{c})]$. It is the average of two normal distribution functions centered at $-c$ and $c$, and there is an inflection for $x>0$ only when $c>1$. Such an inflection point, say $x_{i}$, satisfies the equation $x_{i} / c=\tanh x_{i} c$, where tanh is the hyperbolic tangent. These details are found by setting the second derivative of the function equal to zero. When $c=0$, the function degenerates to $\Phi(x)$ which is the normal S2AFC psychometric function for the ideal detector. The shapes of the function for several values of $c$ are illustrated in Fig. 1.

The same psychometric function is deducible from an assumption of response preferences which could be random in direction so that no consistent preference would be observable from the frequencies of choices. Probably there are other assumptions which would give rise to psychometric functions the same as or very similar to this, and we therefore interpret $c$ merely as a measure of the amount of low-signal suppression. It will be assumed below that $x$ as defined above is approximately proportional to the physical magnitude of the signal; in other words, that the proper psychophysical scale transformation would not change the shape of the psychometric function very much. This is justified for most reasonable physical measures if the signal is small relative to the background to which it is added. A Taylor expansion 


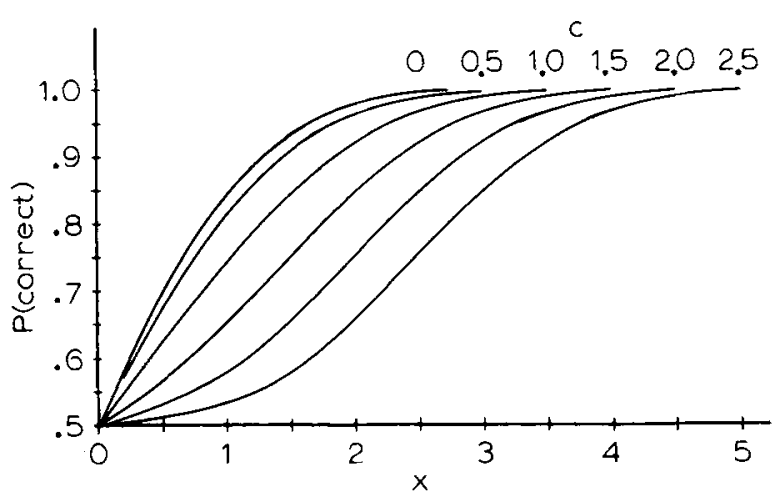

Fig. 1. Effects of different amounts of low-signal suppression according to the proposed psychometric function. Each curve is truncated above at about the .997 point.

about the point at the background level shows the psychophysical transformation to be almost linear within a small range if it is defined for instance by a power function.

To fit the psychometric function to a set of data, it is usually necessary to also estimate a scale parameter. Hence, the psychometric function to be used practically will be denoted by $F(s)$ and defined as

$$
\mathrm{F}(\mathrm{s})=1 / 2\left[\Phi\left(\frac{\mathrm{s}-\mathrm{k}}{\sigma}\right)+\Phi\left(\frac{\mathrm{s}+\mathrm{k}}{\sigma}\right)\right],
$$

where $\mathrm{s}$ is the magnitude of the signal in physical units, $\sigma$ is the scale parameter, and $\mathrm{k}=\sigma \mathrm{c}$. $\mathrm{F}(\mathrm{s})$ is a probability distribution function in $-\infty, \infty$ with a corresponding density function, denoted by $\mathrm{f}(\mathrm{s})$, that is symmetric about the line $\mathrm{s}=0$ and is normal if $k=0$. For $k>0$ the distribution is platykurtic. For $\mathrm{k}>\sigma$ it is bimodal, and it is found that the 75 th percentile is a very close estimate of $k$. The parameter $k$ is roughly a measure of the distance from the origin to the steep part of $F(s)$, and $\sigma$ is an inverse measure of the steepness in that region. For $\mathrm{k}=0$ $\mathrm{s}$ is approximately proportional to $\mathrm{d}^{\prime}$ within a small range, i.e., $s=0 \mathrm{~d}^{\prime} / \sqrt{2}$ approximately for small $s$. For $k>0$ the relationship between $s$ and $d^{\prime}$ is not clear since the definition of $d^{\prime}$ assumes the ideal detector. The estimate of $\sigma$ might be used to plot a theoretical psychometric function for the ideal detector on the assumption that it is in principle possible to reduce $\mathrm{k}$ to 0 .

The moment generating function, $\mathrm{M}(\theta)$, corresponding to the distribution function $F(s)$ is just the average of the moment generating functions of the two normal distributions in terms of which $F(s)$ is defined. It can be expressed as

$$
\mathrm{M}(\theta)=\exp \left(\theta^{2} \sigma^{2} / 2\right) \cosh (\theta \mathrm{k}),
$$

where $\cosh (\theta \mathrm{k})$ is the hyperbolic $\operatorname{cosine}, 1 / 2 \exp (\theta \mathrm{k})+$ $1 / 2 \exp (-\theta \mathrm{k})$. All the odd moments are zero, but the second and fourth moments, $\mu_{2}=\mathrm{k}^{2}+\sigma^{2}$ and $\mu_{4}=\mathrm{k}^{4}+6 \mathrm{k}^{2} \sigma^{2}+\sigma^{4}$, provide the information needed. Usually, there are data only in the half-range, $\overline{0, \infty}$. Therefore it is useful to consider the half-range distribution function, denoted by $G(s)$, defined as $\mathrm{G}(\mathrm{s})=2 \mathrm{~F}(\mathrm{~s})-1$ for $0<\mathrm{s}<\infty$. The corresponding density function is $\mathrm{g}(\mathrm{s})=2 \mathrm{f}(\mathrm{s})$ and the $\mathrm{nth}$ moment is $\int_{0}^{\infty} \mathrm{s}^{\mathrm{n}} \mathrm{g}(\mathrm{s}) \mathrm{ds}=$ $2 \int_{0}^{\infty} \mathrm{s}^{\mathrm{n}} \mathrm{f}(\mathrm{s}) \mathrm{ds}$. By symmetry when $\mathrm{n}$ is even, $\int_{-\infty}^{\infty} \mathrm{s}^{\mathrm{n}} \mathrm{f}(\mathrm{s}) \mathrm{ds}=$ $2 \int_{0}^{\infty} s^{n} f(s) d s$, so that the even moments of $f(s)$ over the full range are the same as the even raw moments of $g(s)$ over the half-range. For half-range distributions such as $g(s)$, there is the identity (McGill, 1963), $\quad \int_{0}^{\infty} \mathrm{s}^{n} \mathrm{~g}(\mathrm{~s}) \mathrm{ds}=$ $n \int_{0}^{\infty} \mathrm{s}^{\mathrm{n}-1}[1-\mathrm{G}(\mathrm{s})] \mathrm{ds}$, which provides a direct method of estimating the moments from tail data. The integral on the right can be approximated by the trapezoidal method from data consisting of an ordered sequence of estimates, $G\left(s_{1}\right)$, $G\left(s_{2}\right) \ldots$ For equal spacing of width $h, s_{i}=i h$, and the trapezoidal approximation can be expressed in terms of a Riemann sum, ${ }^{3}$

$$
\mu_{\mathrm{n}}=2 n h^{\mathrm{n}} \sum_{\mathrm{i}=1}^{\infty} \mathrm{i}^{\mathrm{n}-1} \mathrm{Q}_{\mathrm{i}}, \mathrm{n}>1
$$

where $Q_{i}$ is the relative frequency of wrong responses at the ith signal value. The infinite sum is truncated in practice at some point where $Q_{i}$ becomes sufficiently small. The truncation errors are as small as the corresponding errors in the frequency method of estimating the moments of a distribution, since $n \int_{0}^{x} s^{n-1}[1-G(s)] d s=\int_{0}^{x} s^{n} g(s) d s+$ $x^{n}[1-G(x)]$, which can be verified by differentiation. The spacing or grouping errors are probably of the same orders of magnitude in the two methods.

So far, the assumptions about the nature of the process giving rise to the estimates, $Q_{i}$, have been very weak. For the development of other statistics, it is assumed that the $Q_{i}$ are independent estimates of the corresponding binomial probabilities. This hinges mainly on whether or not the psychometric function remains fixed during the data collection. If the scale parameter, say, were to change slightly from time to time, the $\mathrm{Q}_{\mathrm{i}}$ and other derived statistics would have variances somewhat greater than those based on the assumption of binomial probabilities. The usual efforts to hold conditions constant during data collection are probably sufficient to prevent extreme underestimation of the variances, and useful statements still can be made in terms of inequalities involving confidence regions. The first result of the assumption is $\operatorname{var}\left(Q_{i}\right)=Q_{i}\left(1-Q_{i}\right) /\left(n_{i}-1\right)$, where $n_{i}$ is the number of observations on which $Q_{i}$ is based. Also, from the assumption of independence, $\operatorname{cov}\left(Q_{i}, Q_{j}\right)=0$ for $i \neq j$. The variances and covariances of sums of independent random variables present no problems, and we may write

$$
\operatorname{var}\left(\mu_{n}\right)=4 n^{2} h^{2 n} \sum_{i=1}^{\infty} i^{2 n-2} Q_{i}\left(1-Q_{i}\right) /\left(n_{i}-1\right)
$$

and

$$
\operatorname{cov}\left(\mu_{n}, \mu_{j}\right)=4 n j h^{n+j} \sum_{i=1}^{\infty} i^{n+j-2} Q_{i}\left(1-Q_{j}\right) /\left(n_{i}-1\right) .
$$

The estimates of $\sigma$ and $\mathrm{k}$ are

$$
\mathrm{k}=\left(\frac{3 \mu_{2}^{2}-\mu_{4}}{2}\right)^{1 / 4} \text { and } \sigma=\left[\mu_{2}-\left(\frac{3 \mu_{2}^{2}-\mu_{4}}{2}\right)^{1 / 2}\right]^{1 / 2}
$$

These are nonlinear, but first-order approximations to their variances can be obtained by the method described by Kendal and Stuart (1963, p. 231). They are expressible as

$$
\begin{aligned}
\operatorname{var}(\mathrm{k})=(2 \mathrm{k})^{-6}\left[\operatorname{var}\left(\mu_{4}\right)-12 \mu_{2} \operatorname{cov}\left(\mu_{2}, \mu_{4}\right)\right. & \left.+36 \mu_{2}^{2} \operatorname{var}\left(\mu_{2}\right)\right] \\
\operatorname{var}(\sigma)=(2 \sigma)^{-2}(2 \mathrm{k})^{-4}\left[\operatorname{var}\left(\mu_{4}\right)-4\right. & \left(\mathrm{k}^{2}+3 \sigma^{2}\right) \operatorname{cov}\left(\mu_{2}, \mu_{4}\right) \\
& \left.+4\left(\mathrm{k}^{2}+3 \sigma^{2}\right)^{2} \operatorname{var}\left(\mu_{2}\right)\right] \\
\operatorname{cov}(\sigma, \mathrm{k})=-(2 \sigma)^{-1}(2 \mathrm{k})^{-5}\left[\operatorname{var}\left(\mu_{4}\right)-\right. & \left(8 \mathrm{k}^{2}+6 \sigma^{2}\right) \operatorname{cov}\left(\mu_{2}, \mu_{4}\right) \\
+ & \left.12 \mu_{2}\left(\mathrm{k}^{2}+3 \sigma^{2}\right) \operatorname{var}\left(\mu_{2}\right)\right] .
\end{aligned}
$$

The fourth power of the estimate of $\mathbf{k}$ is minus one-half the fourth cumulant, and there is no strict inequality ensuring that its sampling values must always be positive. In case of a negative estimate, it would be judged on the basis of $\operatorname{var}\left(\mathrm{k}^{4}\right)$, and a significantly negative estimate would be grounds for rejecting the psychometric function proposed here. At the 
other extreme, a special case of the Liapounoff inequality for absolute raw moments (Kendal \& Stuart, 1963, p. 63) is $\mu_{4} \geqslant \mu_{2}^{2}$. Hence, $\mathrm{k}^{2} \leqslant \mu_{2}$ for real $\mathrm{k}^{2}$, and there is no danger of obtaining a negative estimate of $\sigma^{2}$. The standard error of the ratio, $\mathrm{c}=\mathrm{k} / \sigma$, can be approximated by similar methods, and the approximation formula for the variance of the ratio of two random variables is well known (Kendall \& Stuart, 1963, p. 232).

Two experiments were carried out on the detection of a small increment of luminance added to or subtracted from a constant adapting luminance, where the S2AFC task was used in such a way that the above estimates of $\sigma$ and $k$ could be calculated. The experiments were designed to try out the method of estimation, and to investigate the effects on $\sigma, \mathrm{k}$, and $k / \sigma$, of the variation of one experimental parameter, the adapting level.

\section{METHOD}

The experiments were controlled by a DEC PDP-8 laboratory computer interfaced for the control of visual and auditory stimulus sources and for the input and storage of response information from switches operated by the $S$. The visual stimulus was a uniform circular surface with a central dark fixation point. It subtended $25 \mathrm{deg}$ at the eye and was viewed monocularly and centrally against a dark surrounding from a distance of $9 \mathrm{in}$. through a $2-\mathrm{mm}$ artificial pupil, a +4 diopter lens, and neutral density filters. A head rest and bite board with a plaster dental impression were used for reliable positioning of the eye. The 25 deg field was illuminated from the front by two NE-79 neon lamps at $100 \mathrm{~V} \mathrm{dc}$, and the different intensities were obtained by changing the on-time during each $2-\mathrm{msec}$ period of time, so that there was always an intermittency at $500 \mathrm{cps}$ which is far above fusion. The relative effective intensities are then determined by the Talbot-Plateau law, and the basic adapting level was always set at the level given by equal on- and off-times to minimize overlap between the rise and decay curves. The signal to be detected was a luminance increment of the whole $25 \mathrm{deg}$ target, lasting $0.1 \mathrm{sec}$. Neutral density filters were used to give the three different adapting levels, 1, 2, and 4 Trolands. The photometric estimate was made with a Macbeth illuminometer, with the light-adapted eye. The red of the neon source prevented great precision of the measurement, $0.38 \mathrm{ft}-\mathrm{L}$ made at Density 0, but all other photometric quantities are calculated relative to it. A clearly audible $250 \mathrm{cps}$ tone was used to mark the observation intervals all of which were $0.1 \mathrm{sec}$ in length.

Each trial consisted of the events, (a) a response by the $S$, (b) a 1-sec waiting interval, (c) the first observation interval, (d) a 0.75 -sec intersignal interval, (e) the second observation interval, and (f) the time until the S's response. Event (f) of one trial was followed by Event (a) of the next. The $S$ was not informed as to the correctness of his response after each trial. The base adapting luminance level was present at all times except during the observation interval that contained the signal, in order to maintain a constant adaptation level. This was made possible by the use of the program-interrupt facility of the PDP-8, together with a buffered microsecond clock which was interfaced with the computer. Thus, a time-sharing scheme permitted the recording and storage of the response and the lookup of the next signal value without interruption of the adapting level. The microsecond clock interrupted any other activities each time the light was due to be turned on or off. The signal interval was chosen just before each trial on the basis of a random number obtained by the method of Green, Smith, and Klem (1959). The sequence of 10 instances of each of 10 signal values for each run of 100 trials was randomized off-line and read into core storage from paper punch-tape just before the run. Each run took about $5 \mathrm{~min}$, and one session on a given day consisted of 20 runs which took about $2 \mathrm{~h}$. The $S$ adapted to the dark for $20 \mathrm{~min}$ before beginning a session, and then adapted to the adapting luminance of the target for $1 \mathrm{~min}$ before beginning the first run. The $S$ voluntarily blinked after each trial to lower the probability of an involuntary blink during one of the observation intervals. The author, who has no known color anomaly nor refractive error, acted as the $\mathrm{S}$ for all the data reported here.

\section{Experiment 1}

In exploratory experimentation, signals consisting of negative vs positive increments had the same appearance when the magnitude of the signal was small. With signals of greater magnitude, the appearances differed in that the positive increment seemed to be detectable on the basis of either or both of two phenomenal properties. One is just a noticeable "interruption" while the other is a perceptible brightness change. The negative increment seemed to give rise only to the phenomenal interruption, and since the detection task seemed more elementary, negative increments were used as the signals throughout the first experiment.

Data were collected for plotting the psychometric functions at the three adapting levels, 1, 2, and 4 Trolands. A large amount of data was obtained in each case over a period of about a week in order that the shapes of the functions might be unambiguous on inspection.

\section{Experiment 2}

Lest the low-signal suppression obtained in the first experiment be attributed somehow to the use of negative increments for the signals, the 4-Troland condition was repeated using positive rather than negative increments.

\section{RESULTS}

The numerical data for all conditions in both experiments are given in Table 1. The table entries are the relative frequencies of correct response, each based on 1000 trials. Aside from putting the data on record, this table shows that there is not much difference in the psychometric functions for the negative and positive increment at the adapting level of 4 Trolands.

Table 2 lists the estimates, standard errors, and intercorrelations of estimates of the first four moments of the psychometric functions for Experiment 1 . Although only $\mu_{2}$ and $\mu_{4}$ were required for the model considered here, $\mu_{1}$ and $\mu_{3}$ are included in case they may be relevant to some other model. The second moment, or its square root, is probably a good lumped measure of sensitivity, and the three estimates here show the decreasing sensitivity with increasing adapting level, consistent with the general sense of the Weber relation.

Table 1

Raw Estimates of the Probability of Correct Choice at 10 Steps of the Signal Increment in Experiments 1 and 2 with 1/10 Troland as Unit of Measurement

\begin{tabular}{rcccc}
\hline & \multicolumn{5}{c}{ Step/Adapting } & Level \\
Steps & $-.16 / 10$ & $-.24 / 20$ & $-.36 / 40$ & $+.36 / 40$ \\
\hline 1 & .545 & .485 & .514 & .556 \\
2 & .561 & .577 & .534 & .538 \\
3 & .593 & .613 & .602 & .561 \\
4 & .696 & .693 & .687 & .657 \\
5 & .818 & .775 & .789 & .773 \\
6 & .869 & .881 & .863 & .863 \\
7 & .944 & .948 & .929 & .940 \\
8 & .969 & .970 & .974 & .972 \\
9 & .993 & .989 & .989 & .996 \\
10 & .995 & .996 & .997 & .998 \\
\hline
\end{tabular}


Table 2

Estimates and Standard Errors of Raw Moments of the S2AFC Psychometric Function from Experiment 1 with 1/10 Troland as the Unit of Measurement

\begin{tabular}{|c|c|c|c|c|c|c|}
\hline \multirow{2}{*}{$\begin{array}{c}\text { Adapting } \\
\text { Level }\end{array}$} & \multirow[b]{2}{*}{$\mu$} & \multirow{2}{*}{ Estimate } & \multirow{2}{*}{$\begin{array}{l}\text { Standard } \\
\text { Error }\end{array}$} & \multicolumn{3}{|c|}{$\begin{array}{c}\text { Sampling Correlations } \\
\text { of Estimates }\end{array}$} \\
\hline & & & & $\mu_{1}$ & $\mu_{2}$ & $\mu_{3}$ \\
\hline \multirow[t]{4}{*}{40} & $\mu_{1}$ & 1.708 & .0264 & & & \\
\hline & $\mu_{2}$ & 3.408 & .0757 & .871 & & \\
\hline & $\mu_{3}$ & 7.693 & .2342 & .695 & .945 & \\
\hline & $\mu_{4}$ & 18.764 & .7648 & .553 & .843 & .968 \\
\hline \multirow[t]{4}{*}{20} & $\mu_{1}$ & 1.115 & .0174 & & & \\
\hline & $\mu_{2}$ & 1.459 & .0330 & .871 & & \\
\hline & $\mu_{3}$ & 2.179 & .0680 & .688 & .942 & \\
\hline & $\mu_{4}$ & 3.531 & .1499 & .539 & .832 & .966 \\
\hline \multirow[t]{4}{*}{10} & $\mu_{1}$ & 0.725 & .0116 & & & \\
\hline & $\mu_{2}$ & 0.637 & .0150 & .869 & & \\
\hline & $\mu_{3}$ & 0.634 & .0200 & .686 & .942 & \\
\hline & $\mu_{4}$ & 0.685 & .0294 & .538 & .833 & .966 \\
\hline
\end{tabular}

The square root of $\mu_{2}$ has the dimensionality of stimulus units, and the observed values of $\mu_{2}$ are therefore more consistent with the square-root law of DeVries and Rose (McGill, 1967) than with Weber's law.

The high intercorrelations of estimate of the moments are not startling, since these are raw moments. The central moments or cumulants would be less correlated, and a set of four uncorrelated statistics which are linear functions of the raw moments could be obtained by the process of orthogonalization on the basis of the observed variances and covariances.

From Table 2, the estimates and standard errors of $\sigma, \mathrm{k}$, and $\mathrm{k} / \sigma$ were calculated and listed in Table 3 . The ratio, $\mathrm{k} / \sigma$, shows an apparent but slight increase with adapting level. For testing the difference between the values of the ratio for two different adapting levels, a standard error of the difference is obtained as the square root of the sum of the squares of the two individual standard errors. All differences are less in magnitude than two standard errors, and the null hypothesis of invariance is therefore acceptable for this range of adapting levels. As a test of whether or not there was any low-signal suppression, the null hypothesis is $\mathrm{k}=0$ which clearly is rejectable in all cases at very high levels of confidence.

In Fig. 2, the estimates of $\sigma$ and $\mathrm{k}$ were used to plot the continuous theoretical psychometric functions for the data of

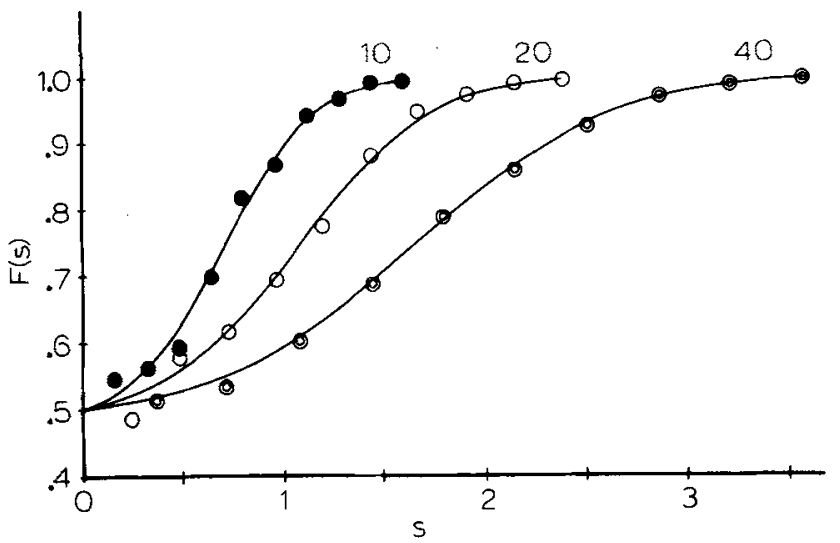

Fig. 2. The S2AFC psychometric functions fitted to the data of Experiment 1. The experimental parameter is the adapting level in units of $1 / 10$ Troland, and the abscissa is the magnitude of the signal increment in the same units.
Experiment 1. The fits to the data are obviously good, whereas the one-parameter normal psychometric function would be too steep near the origin or not steep enough away from the origin.

Table 4 shows that there is as much or more low-signal suppression in the detection of the positive signal increment in Experiment 2, as for the negative in Experiment 1. The parameter estimates differ somewhat in the two comparable cases, but fine comparisons probably are not justified in view of the possibility that our estimates of the standard errors may be a little small for reasons that have been discussed.

Table 3

Estimates and Standard Errors of the Scale and Low-Signal-Suppression Parameters of the S2AFC Psychometric Functions of Experiment 1 with $1 / 10$ Troland as the Unit of Measurement

\begin{tabular}{lcccc}
\hline $\begin{array}{c}\text { Adapting } \\
\text { Level }\end{array}$ & Parameter & Estimate & $\begin{array}{c}\text { Standard } \\
\text { Error }\end{array}$ & $\begin{array}{c}\text { Sampling } \\
\text { Correlation } \\
\text { with } \sigma\end{array}$ \\
\hline 40 & $\sigma$ & .7567 & .0241 & \\
& $\mathrm{k}$ & 1.6839 & .0260 & -.918 \\
& $\mathrm{k} / \sigma$ & 2.2253 & .0919 & \\
\hline \multirow{2}{*}{20} & $\sigma$ & .5140 & .0170 & \\
& $\mathrm{k}$ & 1.0931 & .0176 & -.944 \\
& $\mathrm{k} / \sigma$ & 2.1265 & .0915 & \\
\hline \multirow{2}{*}{10} & $\sigma$ & .3480 & .0114 & \\
& $\mathrm{k}$ & .7183 & .0124 & -.986 \\
& $\mathrm{k} / \sigma$ & 2.0640 & .0906 & \\
\hline
\end{tabular}

\section{DISCUSSION}

There were two main ideas of this study which deserve some review in the light of the collected data.

First, we have proposed-and by our results justified to some extent-a certain two-parameter S2AFC psychometric function in place of the one-parameter zero-centered normal distribution function. What can we offer the sensory psychophysicist to replace the simple threshold measurements of classical psychophysics or the transformation from a proportion of correct responses to a value of d' (Swets, 1964, Appendix 1)? Any general answer must be rather provisional until more is known about the shapes of empirical psychometric functions. If the investigator knows whether or not there is much low-signal suppression in the S2AFC psychometric functions for the class of processes under study, there are simple procedures that seem justifiable. If there is not much, then the transformation to $\mathrm{d}^{\prime}$ will give results which are consistent with one another. If there is much, then as we have seen, the psychometric function resembles that derivable from a certain threshold model where the threshold is the chief source of insensitivity. Table 5 lists the per cent correct

Table 4

Statistics from Experiment 2 with Positive Increment

\begin{tabular}{crrc}
\hline Parameter & Estimate & $\begin{array}{c}\text { Standard } \\
\text { Error }\end{array}$ & $\begin{array}{c}\text { Sampling } \\
\text { Correlation }\end{array}$ \\
\hline$\mu_{2}$ & 3.480 & .0747 & .848 \\
$\mu_{4}$ & 18.240 & .7019 & \\
$\sigma$ & 0.687 & .0228 & -.839 \\
$\mathrm{k}$ & 1.735 & .0248 & \\
$\mathrm{k} / \sigma$ & 2.525 & .1158 & \\
\hline
\end{tabular}


Table 5

Values of the Psychometric Function F(s) at $s=k$ for Different Values of $\mathbf{k} / \sigma$

\begin{tabular}{cc}
$\mathrm{k} / \sigma$ & $\mathrm{F}(\mathrm{k})$ \\
\hline .5 & .6706 \\
.75 & .7166 \\
1.00 & .7387 \\
1.25 & .7419 \\
1.50 & .7493 \\
1.75 & .7499 \\
\hline
\end{tabular}

according to our psychometric function for $\mathrm{s}=\mathrm{k}$ at several values of the ratio $\mathrm{k} / \sigma$. When $\mathrm{k} / \sigma>1$ it is notable that to a close approximation, the model is consistent with the time-honored conventional designation of the 75 th percentile as the threshold. Hence, there might be conceivable occasions to transform to $\mathrm{d}^{\prime}$ and others to estimate the 75 th percentile. From Fig. 2, the interpolated values of the 75 th percentile are found to be very near the numerical estimates of $k$. If this amount of low-signal suppression is typical, then the safest procedure short of estimating both parameters seems to be to stick to the 75 th percentile which is almost a pure measure of $k$. Other percentiles depend jointly on $k$ and $\sigma$ in ways that are not convenient for analysis.

We have proposed secondly a method of fitting the psychometric function. It is based on a method of moments similar to that used for fitting theoretical distributions to grouped-frequency data. Similarly, it is subject to errors of spacing and truncation, and therefore it does not yield exact maximum-likelihood estimates of the parameters. Evidently, these biases were not large with the spacing and ranges of data here; otherwise one would expect obvious discrepancies in the fits to the data. Also in common with the grouped-frequency method of moments, the present method does not presuppose a particular form of the function. The moment estimates obtained here could as well be used to fit some other theoretical function to the data. In principie, this method of moments might be adapted for forced-choice psychometric functions with more than two alternatives, since it requires only that there be prior knowledge of the chance point. However, the theoretical parameters might not be so easily expressible in terms of the moments of the segment of the function above the chance point.

Finally, we have saved another possible source of low-signal suppression until now, because our empirical findings seem to have some bearing. In McGill's (1967) view of sensory energy detection, the sensory excitation distributions are discrete distributions of the Poisson type, the random variates being integer counts of neural impulses. The restriction to integers gives rise to a S2AFC difference threshold somewhat similar to that from which our psychometric function was derived. The thresold corresponds to a single neural impulse which is the least magnitude by which the signal and no-signal counts may differ. In the case of vision, McGill assumed a proportionality constant, a, which is the ratio of the mean impulse count to the number of equal-energy quanta absorbed in the receptor.
A constant proportionality factor would give rise to invariance under changes in the adapting level, of the threshold measured in energy units. On the contrary, approximate invariance was observed here for $\mathrm{k} / \sigma$ which would be the threshold in standardized Thurstone-difference units. This means that the proportionality factor would itself have to be approximately inversely proportional to the square root of the adapting level, i.e., to the ratio of the mean and standard deviation of the adapting-level Poisson quantum count. This alone seems to wreak no havoc with the important properties of the neural counting detector, but another difficulty is the size of $\mathrm{k} / \sigma$ which had observed values of about 2 . Such a value would correspond to $\mathrm{d}^{\prime}=2 \sqrt{2}$ as the detectability for the ideal detector with a simple Gaussian input channel, of the threshold signal for the Poisson detector. The basis for a good guess at the Gaussian $\mathrm{d}^{\prime}$ of the Poisson neural counting unit is not clear, but one certainly would not expect good normal approximations to distributions with such large steps. The proven serviceability of the normal assumption in signal detection work suggests therefore that the low-signal suppression seen here is not all due to the discreteness of the neural-counting distributions.

As for pinpointing the explanation of the effect, this leaves us almost as puzzled as ever. However, new possible sources of it have been pointed out and a basic method for measuring it was developed. Refinements of the method to save observation time can be made in the form of a sequential estimation scheme in which the stimulus values are chosen so as to reduce the standard errors of estimate at a maximal rate.

\section{REFERENCES}

GREEN, B. F., JR., SMITH, J. E. K., \& KLEM, L. Empirical tests of an additive random number generator. Journal of the Association for Computing Machinery, 1959, 6, 527-537.

GREEN, D. M. Auditory detection of a noise signal. Journal of the Acoustical Society of America, 1960, 32, 121-131.

GREEN, D. M., \& SWETS, J. A. Signal detection theory and psychophysics. New York: Wiley, 1966.

KENDALL, M. G., \& STUART, A. The advanced theory of statistics, Vol. 1, (2nd ed.). New York: Hafner, 1963.

McGILL, W. J. Stochastic latency mechanisms. In R. D. Luce, R, R. Bush, and E. Galanter (Eds.), Handbood of mathematical psychology, Vol. 1. New York: Wiley, 1963. Pp. 309-360.

McGILL, W. J. Neural counting mechanisms and energy detection in audition. Journal of Mathematical Psychology, 1967, 4, 351-376.

SWETS, J. A. Is there a sensory threshold? Science, 1961, 134, 168-177.

SWETS, J. A., TANNER, W. P., JR., \& BIRDSALL, T. G. Decision processes in perception. Psychological Review, 1961, 68, 301-340.

SWETS, J. A. (Ed.), Signal detection and recognition by human observers: Contemporary readings. New York: Wiley, 1964.

\section{NOTES}

1. This research was performed while the author was a postdoctoral fellow at the Mental Health Research Institute, supported by NIMH Research Training Grant MH 7417. The author was stimulated by discussions with Drs. Sylvan Kornblum, Irwin Pollack, Harry Gollob, and J. E. Keith Smith although their views are not necessarily reflected accurately here.

2. Now at Cleveland State University.

3. For $\mathrm{n}=1$, add $\mathrm{h} / 2$.

(Accepted for publication September 8, 1968.) 